\title{
EL DERECHO A LA PROPORCIONALIDAD DE LAS MULTAS EN LA JURISPRUDENCIA DE LA SUPREMA CORTE
}

\author{
José María SoBERANES DÍEZ
}

\section{INTRODUCCIÓN}

El presente trabajo tiene como objeto comentar la sentencia dictada por la Suprema Corte de Justicia de la Nación al resolver la acción de inconstitucionalidad 115/2008, el 25 de febrero de 2010, por mayoría de ocho votos.

En dicha acción de inconstitucionalidad, el Procurador General de la República impugnó el artículo 64, último párrafo, de la Ley de Transporte y Vialidad del Distrito Federal, publicado el 30 de septiembre de 2008 en la Gaceta Oficial del Distrito Federal, el cual establece que el titular de una licencia que haya sido cancelada no puede conducir automotores en el territorio del Distrito Federal con licencia o permiso expedido en otra entidad federativa o país, so pena de una multa de 180 días de salario. ${ }^{1}$

Aducía el Procurador que la norma era inconstitucional por preveer una multa fija, esto es, una multa que no contaba con un monto máximo y un mínimo, lo que se traducía en una multa excesiva, de las prohibidas por el artículo 22 constitucional.

Para profundizar sobre la sentencia se analizará, en primer lugar, el marco constitucional acerca de las multas fijas, para lo cual deben distin-

1 Textualmente, los dos últimos párrafos del precepto impugnado establecen: “Asimismo, el titular de la licencia o permiso cancelado queda impedido para conducir automotores en el territorio del Distrito Federal con licencia o permiso expedido en otra entidad federativa o país.

El conductor que sea sorprendido infringiendo el párrafo anterior, se le impondrá una sanción de ciento ochenta días de salario mínimo y se remitirá el vehículo al depósito.” 
guirse dos etapas de la interpretación: el periodo en el que no existe una norma constitucional expresa que mandate la proporcionalidad y, otro, a partir de su establecimiento explícito en una reforma constitucional de junio de 2008. Tratados los aspectos relevantes de las etapas, se abordarán los argumentos esgrimidos en la sesión en la que se dictó la sentencia en comento, así como aquéllos en los que se funda la resolución en análisis.

\section{LA PROPORCIONALIDAD IMPLÍCITA: \\ LO PROPORCIONAL ES LO NO EXCESIVO}

Ante la falta de un mandato constitucional para establecer multas proporcionales, la Suprema Corte sostuvo que las multas fijas eran contrarias al artículo 22 de la Constitución Federal pues, al aplicarse a todos por igual, propicia excesos autoritarios y tratamiento desproporcionado a los particulares. A la luz de este criterio se resolvieron tanto de juicios de amparo como controversias constitucionales y acciones de inconstitucionalidad.

La primera resolución de la "novena época" en que se sostuvo este razonamiento fue el amparo en revisión 2071/93, promovido por Grupo de Consultores Metropolitanos, sociedad anónima de capital variable, resuelto por unanimidad de votos el 24 de abril de $1995 .^{2}$

En ese asunto matriz se partió de la base de que la multa excesiva era una sanción susceptible de ser decretada tanto en materia penal como en administrativa. Se consideró que si la Constitución prohíbe esas sanciones en el ámbito penal, que es el más drástico, con mayor de razón debían quedar prohibidas en los ilícitos administrativos.

Sentado que la Constitución prohíbe las multas excesivas en el ámbito del derecho administrativo sancionador, ese tribunal se enfrentó a la problemática de que la prohibición constitucional no define lo que debe

2 Este es el primer asunto que conoció el Pleno después de la reforma constitucional de diciembre de 1994. Las ideas originales sobre esta cuestión son del Tercer Tribunal Colegiado en Materia Administrativa del Primer Circuito, al resolver sobre la inconstitucionalidad del articulo 76, fracción III, del Código Fiscal de la Federación, el 19 de octubre de 1998, mismo que fue confirmado por el Pleno en la "octava época". Al respecto, Góngora Pimentel, Genaro, El derecho que tenemos: la justicia que esperamos, México, Laguna, 2000, p. 190. 
entenderse por multas excesivas. Al respecto, consideró necesario acudir a los conceptos de proporcionalidad y equidad establecidos en el artículo 31 , fracción IV, de la propia carta federal. A partir de estas nociones, se dijo que una multa es excesiva cuando es desproporcionada a las posibilidades económicas del infractor en relación a la gravedad del ilícito y cuando va más adelante de lo lícito y lo razonable.

En este orden de ideas, sostuvo que una multa puede ser excesiva para unos, moderada para otros y leve para muchos. Por ello, se indicó que para cumplir con este mandato constitucional debía establecerse en la ley la posibilidad de que la autoridad facultada para imponerla, en cada caso, determinase su monto o cuantía, tomando en cuenta la gravedad de la infracción, la capacidad económica del infractor, la reincidencia de éste, en su caso, en la comisión del hecho que la motiva, o cualquier otro elemento del que pueda inferirse la gravedad o levedad del hecho infractor, para así determinar individualmente la multa que corresponda. ${ }^{3}$

A partir de este criterio, el Alto Tribunal consideró que el establecimiento de multas fijas es contrario a estas disposiciones constitucionales, pues al aplicarse a todos por igual, de manera invariable e inflexible, propicia excesos autoritarios y tratamiento desproporcionado a los particulares. ${ }^{4}$

3 Multa excesiva. concepto de. De la acepción gramatical del vocablo "excesivo", así como de las interpretaciones dadas por la doctrina y por la Suprema Corte de Justicia de la Nación para definir el concepto de multa excesiva, contenido en el artículo 22 constitucional, se pueden obtener los siguientes elementos: a) Una multa es excesiva cuando es desproporcionada a las posibilidades económicas del infractor en relación a la gravedad del ilícito; b) Cuando se propasa, va más adelante de lo lícito y lo razonable; y c) Una multa puede ser excesiva para unos, moderada para otros y leve para muchos. Por lo tanto, para que una multa no sea contraria al texto constitucional, debe establecerse en la ley que la autoridad facultada para imponerla, tenga posibilidad, en cada caso, de determinar su monto o cuantía, tomando en cuenta la gravedad de la infracción, la capacidad económica del infractor, la reincidencia, en su caso, de éste en la comisión del hecho que la motiva, o cualquier otro elemento del que pueda inferirse la gravedad o levedad del hecho infractor, para así determinar individualizadamente la multa que corresponda (tesis P./J. 9/95, visible en el Semanario Judicial de la Federación y su Gaceta, novena época, julio de 1995, t. II, p. 5).

4 Multas fijas. Las leyes que las establecen son inconstitucionales. Esta Suprema Corte ha establecido que las leyes, al establecer multas, deben contener las reglas adecuadas para que las autoridades impositoras tengan la posibilidad de fijar su monto o cuantía, tomando en cuenta la gravedad de la infracción, la capacidad económica del infractor, la reincidencia de éste en la conducta que la motiva y, en fin, todas aquellas circunstancias 
En este razonamiento se vislumbra que la proporcionalidad es criterio informador de la noción de multa excesiva. Aunque no lo dijo la Corte, puede estimarse que la prohibición constitucional del establecimiento de multas excesivas conlleva implícito un mandato de instituir multas proporcionales.

En efecto, la excesividad es un concepto relacional en tanto que únicamente se entiende en concordancia con algo. Una sanción no es excesiva consigo misma, sino que se entiende que tiene este carácter solamente cuando sobrepasa una medida. Lo excesivo evoca la falta de condición de proporcionalidad de un ente respecto a otro en un margen: mientras que exceso es lo que sobrepasa un límite superior de la medida, lo proporcional es lo que se ajusta a ésta. Por ello, lo contrario a la excesividad es la proporcionalidad.

Así pues, la Constitución Federal, al prohibir la multa excesiva, implícitamente está mandando que se trate de forma proporcional, en tanto esta última noción es antípoda de la primera. De esta forma, el artículo 22 constitucional contiene implícitamente el principio de proporcionalidad de las multas.

\section{LA PROPORCIONALIDAD EXPLÍCITA EN LA CONSTITUCIÓN}

No obstante la existencia implícita del principio de proporcionalidad en las multas desde el texto original de 1917, en el contexto de la reforma constitucional al sistema de justicia penal de junio de 2008, se estableció expresamente que "toda pena deberá ser proporcional al delito que sancione y al bien jurídico afectado". Ésta es la norma en vigor. ${ }^{5}$

que tiendan a individualizar dicha sanción; obligación del legislador que deriva de la concordancia de los artículos 22 y 31, fracción IV, de la Constitución Federal, el primero de los cuales prohíbe las multas excesivas, mientras el segundo aporta el concepto de proporcionalidad. El establecimiento de multas fijas es contrario a estas disposiciones constitucionales, por cuanto al aplicarse a todos por igual, de manera invariable e inflexible, propicia excesos autoritarios y tratamiento desproporcionado a los particulares (tesis P./J. 10/95, visible en el Semanario Judicial de la Federación y su Gaceta, novena época, julio de 1995, t. II, p. 19).

5 Si bien el Artículo Segundo Transitorio de la reforma constitucional establece que la reforma entrará en vigor al publicarse la legislación secundaria, la cual no podrá exceder en realizarse el plazo de ocho años, estimamos que esa vacatio legis es para el sistema procesal y no para los derechos fundamentales que se establecieron en la reforma. 
Aún no existe un desarrollo jurisprudencial de este precepto. Estimamos que el mismo podría partir del hecho que si bien éste se refiere al derecho penal, es posible considerar que es aplicable a las otras materias, como la administrativa. A las razones esgrimidas en el primer asunto que se resolvió para este objeto, que se simplifican en el aforismo "lo que rige a lo más rige a lo menos", puede añadirse la reciente consideración de que los principios del derecho penal son aplicables al derecho administrativo.

El alto tribunal ha sostenido que los principios del derecho penal son aplicables al derecho administrativo sancionador en tanto ambos son manifestaciones del ius puniendi del Estado. ${ }^{6}$ Siguiendo esta línea de argumentación, todas las sanciones administrativas se rigen por los principios constitucionales penales, como lo es el de la proporcionalidad en las sanciones. Así pues, toda sanción administrativa como las multas, la clausura, el arresto administrativo, la inhabilitación, apercibimiento, suspensión, etcétera, debe ser proporcional para cumplir con los estándares constitucionales.

Señalado lo anterior, debería atenderse a la sanción específica en análisis, para lo que tendría que responderse la pregunta a la que se enfrentó el Tribunal Pleno al resolver el citado amparo en revisión 2071/93: ¿qué debe entenderse por una multa proporcional? Esta duda debe contestarse en dos horizontes. Por una parte, en la aplicación de la ley y, por otra, en la formulación de la misma.

En cuanto a la proporcionalidad en la imposición de multas, cabe apuntar brevemente que consiste en la obligación de la autoridad encargada de aplicar esta sanción de motivar su monto de acuerdo a las circunstancias personales del infractor, tomando en cuenta su capacidad económica, la reincidencia o cualquier otro elemento del que se desprenda la levedad o gravedad de la infracción.

Además, dicho transitorio no sujeta al artículo 22 constitucional a dicho plazo, como hace con los otros preceptos reformados.

6 Jurisprudencia P./J. 99/2006, de rubro "derecho administrativo sancionador. Para la construcción de sus propios principios constitucionales es válido acudir de manera prudente a las técnicas garantistas del derecho penal, en tanto ambos son manifestaciones de la potestad punitiva del estado", publicada en el Semanario Judicial de la Federación y su Gaceta, novena época, agosto de 2006, t. XXIV, p. 1565. 
En lo referente a la proporcionalidad en el establecimiento de las multas, igualmente ésta debe entenderse en dos vertientes: desde un punto de vista material y uno formal.

Respecto al punto de vista material, debe decirse que la relación de proporción que debe guardar un comportamiento ilícito con la multa que se le asigne será el fruto de un complejo análisis que sólo al legislador corresponde y que en ningún caso se reduce a una exacta proporción entre el valor de la multa y el del comportamiento prohibido según un hipotético baremo prefijado. No obstante el amplio margen de maniobra del legislador en este aspecto, tiene límites constitucionales. En concreto, no pueden existir normas en las que se advierta un desequilibrio patente, excesivo o irrazonable entre la multa y la finalidad de la norma.

En relación con la variante formal del principio de proporcionalidad en el establecimiento de multas, debe decirse que éste impone la obligación de fijar una multa máxima y una mínima, como presupuesto para que el aplicador de la sanción pueda individualizarla al caso concreto del infractor, eligiendo la multa adecuada dentro de ese rango.

En diversos precedentes la Suprema Corte ya se ha pronunciado sobre este tema, considerando que para que una multa sea acorde al texto constitucional debe contener un parámetro establecido en cantidades o porcentajes mínimos y máximos que permitan a las autoridades que las impongan determinar su monto de acuerdo a las circunstancias personales del infractor, tomando en cuenta su capacidad económica, la reincidencia o cualquier otro elemento del que se desprenda la levedad o gravedad de la infracción. ${ }^{7}$

7 Al respecto es aplicable la jurisprudencia P./J. 102/99: "Multas. las leyes que las establecen en porcentajes determinados entre un mínimo y un máximo, no son inconstitucionales. Esta Suprema Corte ha establecido, en la tesis de jurisprudencia P./J. 10/95, que las leyes que prevén multas fijas resultan inconstitucionales por cuanto al aplicarse a todos por igual de manera invariable e inflexible, propician excesos autoritarios y tratamiento desproporcionado a los particulares; sin embargo, no pueden considerarse fijas las multas establecidas por el legislador en porcentajes determinados entre un mínimo y un máximo, porque con base en ese parámetro, la autoridad se encuentra facultada para individualizar las sanciones de conformidad con la gravedad de la infracción, la capacidad económica del infractor, la reincidencia o cualquier otro elemento del que pueda inferirse la levedad o la gravedad del hecho infractor" (Semanario Judicial de la Federación y su Gaceta, novena época, t. X, noviembre de 1999, p.31.); y la jurisprudencia P./J. 17/2000: "multas. no tienen el carácter de fijas las establecidas en preceptos que prevén una sanción mínima y una máxima. El establecimiento de multas fijas es contrario a los 
De acuerdo a lo expuesto podría llegarse a la misma conclusión a la que se abordó al analizar la prohibición de multas excesivas, en el sentido de que las multas fijas son contrarias al principio constitucional de proporcionalidad en las mismas, en el aspecto formal de su vertiente legislativa.

\section{El DEBATE DE LA Suprema CORTE}

Como se dijo, en la acción de inconstitucionalidad 115/2008, el Procurador General de la República impugnó el artículo 64, último párrafo de la Ley de Transporte y Vialidad del Distrito Federal, al considerar que era inconstitucional la multa fija de 180 días de salario que ahí se establecía.

El proyecto de sentencia fue elaborado por la ministra Luna Ramos y en él se proponía la inconstitucionalidad de la norma, con base en los precedentes antes relatados. El proyecto fue rechazado por mayoría de ocho votos, mismos que consideraron constitucional la multa. ${ }^{8}$

En la sesión en la que se discutió el asunto se divisaron dos posturas. La primera posición era de los que estaban a favor de un cambio de criterio. En esta línea, el ministro Franco González-Salas manifestó que veía prácticamente imposible que el policía tenga que valorar la gravedad de una infracción que es igual para todos, afirmando que habría más discrecionalidad en dejar a juicio de un agente de tránsito que juzgue estas con-

artículos 22 y 31, fracción IV, de la Constitución, por cuanto que al aplicarse a todos los infractores por igual, de manera invariable e inflexible, propicia excesos autoritarios y tratamiento desproporcionado a los particulares. En virtud de ello, los requisitos considerados por este Máximo Tribunal para estimar que una multa es acorde al texto constitucional, se cumplen mediante el establecimiento, en la norma sancionadora, de cantidades mínimas y máximas, lo que permite a la autoridad facultada para imponerla, determinar su monto de acuerdo a las circunstancias personales del infractor, tomando en cuenta su capacidad económica y la gravedad de la violación" (Semanario Judicial de la Federación y su Gaceta, novena época, marzo de 2000, t. XI, p. 59).

8 Votaron por la constitucionalidad los ministros Aguirre Anguiano, Cossío Díaz, Luna Ramos, Franco González-Salas, Zaldívar Lelo de Larrea, Aguilar Morales, Sánchez Cordero y Silva Meza. Por la inconstitucionalidad, los ministros Gudiño Pelayo, Valls Hernández y Ortiz Mayagoitia. 
diciones en la detención, establecer la capacidad económica del sujeto, o determinar si es reincidente para imponer una pena. ${ }^{9}$

El ministro Aguilar Morales abundó en esas consideraciones y manifestó que tratándose de multas administrativas de policía, como las infracciones de tránsito, en la mayoría de los casos dada la inmediatez entre la infracción y la imposición de la sanción no sólo es materialmente imposible fijar parámetros objetivos verificables respecto del infractor, sino que en este tipo de infracciones no podría hacerse una valoración de la mayor o menor trascendencia económica del asunto, pues no existe un referente de esa naturaleza en la infracción cometida, ya que ni el valor del automóvil ni la capacidad económica del infractor son determinantes ni aun relacionados con la objetiva conducta ilícita que se sanciona. ${ }^{10}$

El ministro Zaldivar Lelo de Larrea, en esa misma línea, sostuvo que no debe cumplirse la proporcionalidad formal cuando se está en presencia de cierta conducta "en donde estaríamos ya exigiendo una especificidad excesiva al legislativo, o cuando queda clara la gravedad de la conducta." En el caso le pareció que la conducta era lo suficientemente grave como para que no admita otro tipo de atemperamentos. ${ }^{11}$

En síntesis, en esa posición tiene cuatro bases: a) la infracción es la misma para todos; b) no pueden distinguirse los casos de mayor trascendencia económica, porque no existe un referente de esa naturaleza en la infracción; c) no debe haber gradación en los casos en los que no pueda exigírsele al legislador que distinga varios supuestos de hecho o que la conducta sea muy grave; y d) los policías no pueden juzgar las diferencias en el momento de la detención

Con base en estos argumentos deben señalarse algunas cuestiones. En cuanto a que la infracción es la misma para todos, debe señalarse que, aunque la conducta sea la misma, existen circunstancias que hacen más o menos grave la infracción. Aunque una infracción de tránsito se valore conforme a la justicia conmutativa, viendo los hechos sin importar su autor, debe estimarse que hay circunstancias que afectan al hecho.

9 Versión estenógráfica de la sesión del Pleno de la Suprema Corte correspondiente al 25 de febrero de 2010, visible en http://www.scjn.gob.mx/ActividadJur/Pleno/VerEstenograficas/Paginas/2010.aspx

10 Idem.

11 Idem. 
Hay incidentes que permiten señalar que hay distintas gravedades en las infracciones de tránsito. No es lo mismo pasarse un alto en una calle intransitada que en el cruce de las avenidas con más circulación. En el caso concreto también: no es lo mismo el que tenía una licencia de otra entidad con anterioridad a la del Distrito Federal, que ir a tramitar una licencia a otra a propósito debido a la cancelación de la correspondiente a la ciudad de México.

Respecto a que no existe un referente económico, debe apuntarse que la mayor cuantía de una multa no debe relacionarse únicamente con una cuestión de capacidad contributiva. Es cierto que las multas fiscales suelen establecerse en relación con el monto de la contribución omitida, pero las multas administrativas son más amplias. Su mayor cuantía es en relación con distintas circunstancias que califican la gravedad de la infracción y su lesión al bien jurídico protegido.

En cuanto a que no debe haber gradación cuando no pueda exigírsele al legislador que distinga varios supuestos de hecho o cuando la conducta sea muy grave, conviene apuntar, en primer lugar, que afirmar que cuando una infracción es muy grave no es necesario establecer parámetros, implica desconocer el contenido esencial del principio de proporcionalidad. Supondría afirmar que la gravedad de una conducta justificaría la inaplicación de los derechos fundamentales. Por otra parte, es erróneo asegurar que hay veces que no se le puede exigir al legislador que valore varias circunstancias, pues quien las valora es la administración, siendo obligación del legislador únicamente dar un máximo y un mínimo para que pueda fijarse la multa. La ley no tiene que preveer que pasará si la licencia de otra entidad se obtuvo después; eso lo valora el aplicador.

El argumento práctico, que es difícil que un agente de tránsito valore las circunstancias, parecería alejado de la idea constitucional, pues podría pensarse que en el fondo supone que cuando es complicado aplicar la Constitución, no debe de hacerse. No obstante, considerando que los derechos no son absolutos, habría al menos que darle cabida en el examen de constitucionalidad. Por lo anterior se estima que no son atendibles tres de los argumentos mencionados por esta posición. Únicamente el argumento fáctico, el de la imposibilidad de que el policía valore la gravedad, podría tener relevancia.

La segunda posición la sostuvieron los que estaban de acuerdo con el criterio tradicional. El ministro presidente Ortiz Mayagoitia, sostuvo que 
no es lo mismo un conductor que vive de su trabajo, que alguien que por necesidad acepta conducir un vehículo que va a pasar por la ciudad de México y que se la "juega" y es descubierto. ${ }^{12}$

Asimismo, el ministro Gudiño Pelayo sostuvo que existen elementos que pueden ayudar a determinar un monto distinto para cada caso, como es la edad, el grado de preparación, el modo de vida o las circunstancias particulares que en cada caso concreto se presentan. ${ }^{13}$

Esta postura se basa en que existen diversos elementos que podrían servir para calificar la gravedad de la multa cuya constitucionalidad se cuestionaba, como las circunstancias personales de los infractores, el fin de la conducta, o accidentes que pueden aumentar o atenuar la responsabilidad.

Esta posición se apega más a los mandatos que emanan del principio constitucional de proporcionalidad en las multas. Sin embargo, no toma en cuenta las cuestiones fácticas que son rescatables de la postura anterior. Ciertamente éstas no pueden condicionar la eficacia constitucional $\mathrm{y}$ menos de un derecho fundamental, pero deben ser tomadas en cuenta en el análisis de constitucionalidad.

\section{LA SENTENCIA DE LA ACCIÓN \\ DE INCONSTITUCIONALIDAD 115/2008}

Como se dijo, el proyecto de la ministra Luna Ramos fue rechazado por mayoría de ocho votos. Sin embargo, en lugar de turnar el asunto a un nuevo ponente para que redactara otro proyecto, se estimó que existía una coincidencia entre los integrantes de la mayoría que hacía que hubiera una decisión sobre el tema, por lo que se declaró resuelto el asunto.

La sentencia redactada con posterioridad retoma las ideas expresadas por los ministros en la discusión. En concreto, son dos los argumentos en que se basa la resolución:

La primera razón es que la insistencia del conductor en volver a manejar vehículos en el Distrito Federal, no obstante que su licencia o permiso fue cancelado, lo hace acreedor a una sanción mayor a previa (la cancelación); consistente en la imposición de una multa fija, la cual en estos peculiares casos no requiere de un mínimo y un máximo para graduarla,

12 Idem.

13 Idem. 
ya que las características personales del desobediente en nada influirían para determinar la gravedad o levedad de su conducta.

El segundo motivo para estimar que la multa controvertida no requiere de un mínimo y un máximo para estimarla apegada al artículo 22 constitucional, se encuentra en razones de índole práctico acaecidas en el momento en que se aplica la sanción, ya que la conducta que se describe como el supuesto normativo que da lugar a la imposición de dicha multa, se suscita generalmente en la vía pública y en situaciones de flagrancia, siendo un hecho notorio que los servidores públicos facultados para detectar este género de faltas, en muchos casos, no cuentan con los elementos técnicos o fácticos necesarios que les permitan, en el instante mismo de la comisión de la infracción, la posibilidad de valorar en cada caso la gravedad de aquélla, la capacidad económica del sujeto sancionado y la posible reincidencia de éste en la conducta que la motiva.

En cuanto al primer argumento, de que no es una sanción autónoma y que las características personales no afectan en la gravedad, conviene apuntar que si bien el ilícito no lo determinan circunstancias de la persona, éstas si pueden modificar la gravedad o el monto de la multa. El lugar de residencia o la profesión del infractor pueden hacer más o menos grave la infracción. Como se dijo en la sesión, no es lo mismo el que cruza la ciudad de México, que el que reside en ella. Además no todas las circunstancias que califican la gravedad son personales. Hay incidencias de otro tipo, como el momento en que se obtuvo la licencia, que no tienen cabida con una multa fija.

Respecto al segundo argumento, como se dijo: las cuestiones prácticas en principio no deberían condicionar la eficacia de un derecho fundamental. El legislador o la administración deberían establecer procedimientos de sanción que respeten los derechos. No obstante, estimamos que pudo abordarse en el examen de constitucionalidad. En concreto, pudo haber sido tomada en cuenta en una prueba de razonabilidad.

El derecho a la proporcionalidad en las multas, como todo derecho fundamental, no es absoluto. Pueden existir motivos que justifiquen una interferencia en el rango de actuación del derecho por parte del legislador, siempre y cuando sean razonables, como han sostenido la mayoría de las jurisdicciones constitucionales. Para valorar esta cuestión se utili- 
za un cuestionario, en el que se analiza si existe una razón que justifique si ésta es adecuada y proporcional. ${ }^{14}$

En el caso, el elemento fáctico pudo ser utilizado como fin: no existe gradación para evitar la discrecionalidad de los oficiales de tránsito en tanto no tengan elementos que les permitan valorar la gravedad de la infracción. El medio utilizado sí es adecuado para ese fin, pues si existe una sola multa, el policía no puede distinguir. Por ello, la medida es adecuada.

No obstante, no es proporcional la interferencia del legislador en el derecho fundamental, puesto que la restricción que hace no se limita al caso de la detención de un policía en la calle, sino que también afecta a los casos en que se impugne la multa ante un órgano jurisdiccional.

En efecto, todas las multas de tránsito que se impongan son impugnables ante el Tribunal Contencioso Administrativo del Distrito Federal. La Sala que conozca del asunto sí tiene a su disposición todos los elementos que le permiten valorar las circunstancias que se han mencionado previamente, y que podrían servir como base para disminuir el monto por la atenuación de la responsabilidad, por ejemplo. Sin embargo, dicho órgano no tendría posibilidad legal de modificar el monto de la multa.

Por estas razones, la medida no guarda una relación razonable con el fin que se procura alcanzar; es decir, es más grande la intromisión al derecho que las ventajas que se obtienen con la medida. De esta forma, la multa fija del caso no supera la prueba de razonabilidad y, por tanto, no puede considerarse como una interferencia constitucional al derecho fundamental a la proporcionalidad de las multas.

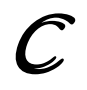

14 No se considera el análisis de la necesidad, es decir, si podría valorarse si existen medidas igualmente de eficaces que restrinjan menos el derecho fundamental, pues la determinación de multas de tráfico es una cuestión en la que el legislador tiene amplia libertad de configuración, lo que hace que el cuestionario de razonabilidad sea más laxo, es decir, que no haga el análisis de necesidad, bajo los parámetros establecidos por la Suprema Corte en la jurisprudencia 1a./J. 84/2006 de rubro "Análisis constitucional. u intensidad a la luz de los principios democrático y de división de poderes", Semanario Judicial de la Federación y su Gaceta, novena época, noviembre de 2006, t. XXIV, p. 29. 